\title{
Atualização sobre tratamento medicamentoso de infecçóes causadas por Staphylococcus aureuse resistência a drogas antiestafilocócicas
}

\section{Update on treatment of infections caused by Staphylococcus aureus and drug resistance to ant staphylococcal drugs}

\author{
Manuela Ornelas de Abreu ${ }^{1,3}$ \\ Claudete Araujo Cardoso ${ }^{2,3}$ \\ Aline Cardoso Caseca Volotão $0^{3,4}$ \\ Fábio Aguiar-Alves*1,3,4
}

\begin{abstract}
Resumo
Desde os primeiros casos de resistência a penicilina e depois meticilina,o Staphylococcus aureusse tornou de grande importância no ambiente hospitalar e atualmente também na comunidade. Glicopeptídeos são a primeira linha de tratamento para infecções graves por MRSA, porém a alta toxicidade dos mesmos e, resistência, fazem com que as outras alternativas terapêuticas sejam adotadas na prática clínica para o tratamento de infecções causadas por $S$. aureus. Esta revisão pretende abordar as principais opções de tratamento antiestafilocócicos.

Palavras chave: Staphylococcus aureus resistente a meticilina, tratamento e infecção.
\end{abstract}

\begin{abstract}
Since the first cases of resistance to penicillin and methicillin, Staphylococcus aureus has become of great importance in the hospital and now also in the community environment. Glycopeptides are the first line of treatment for severe infections caused by MRSA, but the high toxicity and development of resistance induce the adoption of other therapeutic alternatives in clinical practice for treating infections caused by S. aureus. This review aims to address the characteristics of antimicrobial resistance of $S$. aureus and the main of pharmacological treatment options.

Key words: Methicillin-Resistant Staphylococcus aureus, treatment and, infection.
\end{abstract}

\footnotetext{
'Programa de PósGraduação em Patologia - Faculdade de Medicina - Universidade Federal Fluminense - Niterói - Brasil.

${ }^{2}$ Programa de PósGraduação em Ciências Médicas - Faculdade de Medicina - Universidade Federal Fluminense - Niterói - Brasil.

${ }^{3}$ Laboratório Universitário Rodolfo Albino (LURA EX) - Universidade Federal Fluminense - UFF.

${ }^{4}$ Pólo Universitário de Nova Friburgo - Universidade Federal Fluminense - Nova Friburgo - Brasil.
} 


\section{INTRODUÇÃO}

Bactérias descritas no gênero Staphylococcus são classificadas comoGram positivas, dispostas em forma de cocos e geralmente agrupadas em formatos irregulares semelhantes a cachos de uva. São capazes de produzir peróxido de hidrogênio na presença de oxigênio, sendo caracterizadas como catalase positivas (SAntos, SAntos et al. 2007; KateEte, Kimani et al. 2010). Este gênero possui 33 espécies, porém somente 17 podem ser isoladas de material biológico humano (SAntos, SAntos et al. 2007).

A espécie mais patogênica deste gênero é Staphylococcus aureus (Murray, Baron et al. 2003; Kateete, Kimani et al. 2010). Este patógeno pode ser encontrado colonizando $27 \%$ dos seres humanos de maneira assintomática (Young, Golubchik et al. 2012), e os sítios de maior prevalência são fossas nasais, axilas e períneo (GIAROLA, SANTos et al. 2012). Tal agente pode causar diversos processos patológicos, desde mais simples como furúnculo e acne, até mais complexos como sepse, síndrome de choque tóxico, endocardites, pneumonias e infecçôes associadas com dispositivos intravasculares, corpos estranhos e formação de biofilme (Santos, Santos et al. 2007; Hagihara, UMEMURA et al. 2012; RUDKIN, EdWARDs et al. 2012).

O tratamento para doenças estafilocócicas teve início em 1942, 14 anos após a descoberta da penicilina por Alexander Fleming. A penicilina é um composto químico que contém um grupo farmacofórico chamado anel beta-lactâmico (figura 01). Logo após o início da utilização das penicilinas, casos de resistência a mesma foram relatados (Rammelkamp and Maxon 1942). Como alternativa de tratamento para as infecçôes resistentes a penicilina, surgiu a meticilina, uma penicilina modificada, porém dois anos após o uso da meticilina surgiram cepas de estafilococos resistentes, denominadas Staphylococcus aureus Resistentes a Meticilina (MRSA) (Rammelkamp and Maxon 1942; DeRESINSKI 2005; IPPOLITO, LeONE et al. 2010).

Figura 01: Estrutura molecular da Penicilina com grupo farmacofóricobeta-lactâmico.

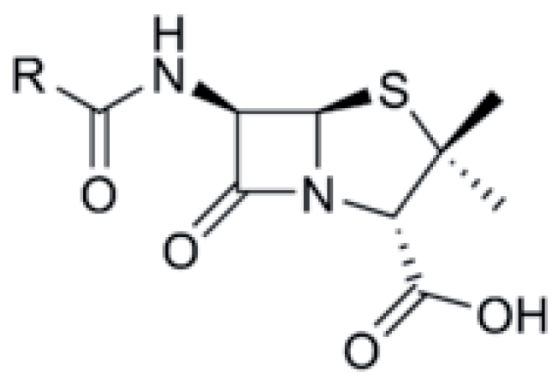

Fonte: Osiris Property Explorer
Essas cepas MRSA limitam o arsenal terapêutico e aumentam o risco de infecçóes de difícil tratamento nos ambientes hospitalares e de comunidade, sendo necessário o conhecimento das opçóes de tratamento de escolha e medidas de prevenção, afim de que seja otimizado o uso racional de antimicrobianos, prevenindo infecçóes e limitando os casos de resistência.

O presente estudo abordará as alternativas de medicamentos existentes para o tratamento de doenças causadas por $S$. aureus, incluindo MRSA e novas alternativas de tratamento para infecçóes por S.aureus devido à resistência a drogas antiestafilocócicas.

\section{STAPHYLOCOCCUS AUREUS: CARACTERÍSTI- CAS}

S. aureus é um dos patógenos mais prevalentes em infecçóes relacionadas à assistência à saúde, por este motivo, diversos estudos têm sido realizados a fim de aprimorar o entendimento dos mecanismos de transmissão e controle de disseminação. Tal transmissão pode ocorrer por fômites (objetos ou superfícies contaminadas), contato com profissionais de saúde colonizados, secreção nasal e, principalmente, o contato direto com lesóes abertas em pacientes infectados (Archer, Mazaitis et al. 2011).

S. aureus possui fatores de virulência específicos e uma capacidade adaptativa que o permite sobreviver a grande variedade de condiçóes ambientais. É capaz de resistir à imunidade inata do organismo e adquiriu mecanismos de resistência a antimicrobianos (FALORD, MADER et al. 2011).

Infecçôes causadas por $S$. aureus, antes da introduçáo de antimicrobianos na prática clínica, levavam a óbito cerca de $80 \%$ da população e $70 \%$ desenvolviam infecçóes metásticas (DERESINSKI 2005).

Infecçóes por MRSA acarretam maior mortalidade, maior tempo de hospitalização e aumento dos custos quando comparados a infecçóes por Staphylococcus aureus Sensível a Meticilina (MSSA) (Hagihara, Umemura et al. 2012).

Cepas MRSA causam graves infecçóes tanto em ambiente hospitalar quanto comunitário. $\mathrm{O}$ aumento da prevalência de infecçôes por MRSA aumenta o uso de glicopeptídeos como a vancomicina para o seu tratamento, com isso dois mecanismos de resistência já foram descritos na evasão deste microorganismo ao tratamento com vancomicina (Climo, Patron et al. 1999; Fox, Lampen et al. 2006). O primeiro, descrito no Japáo em 1996, é caracterizado como um espessamento da parede celular (Hiramatsu, Hanaki et al. 1997) e o segundo, descrito nos EUA em 2002, é um mecanismo que envolve os genes vanA alterados, mo- 
dificando o sítio de ação da vancomicina, mecanismo este semelhante aos de enterococos ((CDC) 2002).

$\mathrm{O}$ combate às cepas de bactérias resistentes aos antibióticos e quimioterápicos se torna consideravelmente difícil, devendo-se adotar medidas que levem à reduçáo do risco de desenvolvimento dessas cepas, aliando um tratamento criterioso dos pacientes com a orientação dos profissionais de saúde e também a capacitação e treinamento adequado dos mesmos, no sentido de que cumpram a posologia da droga e o tempo do tratamento estabelecido na sua totalidade. Paralelamente a isto, medidas complementares devem ser assumidas pelas autoridades responsáveis pelo controle da saúde pública, no desenvolvimento de condiçóes sanitárias apropriadas e nos investimentos em pesquisas científicas que visem à descoberta de novos e mais eficientes antimicrobianos, quimioterápicos e vacinas, para combater as bactérias multirresistentes em particular, e os agentes infecciosos como um todo (Lindsay and Holden 2004).

\section{TRATAMENTO}

O tratamento para infecçôes bacterianas deve ser orientado com conhecimento dos fatores locais, prováveis fontes de infecção e, também prévia análise de fatores de risco relacionados com os pacientes de um hospital ou o meio em que eles vivem. Torna-se necessário um estudo prévio da situação e levantamento de dados epidemiológicos sobre a incidência local. Além disso, cepas resistentes devem ser adotadas para auxiliar na escolha da terapia inicial. Para a escolha da terapia definitiva, exames laboratoriais de identificação do patógeno e testes fenotípicos de resistência como teste de sensibilidade a antimicrobianos são necessários, para que a terapêutica seja direcionada com exatidão, fazendo uso do exato antimicrobiano cujo a cepa analisada seja susceptível. Evitando o uso indiscriminado de antimicrobianos, diminuindo o aumento dos casos de resistência. (Luna, Rodriguez-Noriega et al. 2010).

$\mathrm{O}$ arsenal terapêutico para tratamento de infecçóes estafilocócicas inclui agente tópico, formas farmacêuticas administradas por via oral (VO) e via intravenosa (IV) (SHITtu AO, Udo EE et al. 2009; Luna, Rodriguez-Noriega et al. 2010).

A mupirocina é um agente antimicrobiano de uso tópico, inicialmente isolado de Pseudomonas fluorescens (PARK, KIM et al. 2012). Tal fármaco é utilizado para o tratamento de infecçóes de pele e feridas de pós-operatório. Em crianças, a mupirocina a $2 \%$ é utilizada para tratar infecçóes de pele mais localizadas como impetigo e infecçóes secundárias como eczema, úlceras ou laceraçóes (LiU, BAYER et al. 2011). A mupirocina também é utilizada para descoloni- zação da mucosa nasal por cepas de $S$. aureus. Entretanto, o uso extensivo deste medicamento é denominado como fator de risco para a resistência bacteriana a mupirocina, já detectada anteriormente (ShitTu AO, Udo EE et al. 2009; Rodriguez-Noriega and Seas 2010; Park, Kim et al. 2012). Shittu e colaboradores, em 2009, identificaram altos índices de resistência de $S$. aureus a mupirocina na Nigéria e África do Sul.

Cepas resistentes a mupirocina classificam-se em dois grupos quando analisamos o MIC (Concentração Mínima Inibitória): baixo nível de resistência $(\mathrm{MIC}=8$, aproximadamente $256 \mu \mathrm{g} / \mathrm{mL})$ e alto nível de resistência $(\mathrm{MIC}>256 \mu \mathrm{g} / \mathrm{mL})$ (PARK, Kim et al. 2012).

O tratamento de abscessos simples ou furúnculos limita-se a incisão e drenagem dos mesmos. Caso a infecção seja mais grave ou invasiva, com sintomatologia sistêmica associada (como febre, calafrios e celulite perilesional por exemplo), torna-se necessário o início da antibioticoterapia (Liu, BAYer et al. 2011).

O tratamento de infecçóes por MSSA em pacientes clinicamente estáveis ocorre com a utilização via oral de cefalosporinas de primeira geração como a cefalexina ou cefadroxil. Em casos mais graves faz-se necessário uma terapia parenteral com oxacilina. A duração da terapia depende do foco da infecção e pode durar por no mínimo 10 dias, podendo ser prolongada usualmente até 21 dias, dependendo da evolução clínica do paciente (DA Costa, Pinheiro et al. 2011).

Para cobertura empírica de infecções por $S$. aureus que não respondem a beta-lactâmicos ou demonstram sinais de toxicidade, a opção por via oral pode ser clindamicina, trimetropim-sulfametoxazol ou linezolida (LiU, BAYER et al. 2011).

Vancomicina é um glicopeptídeo empregado no tratamento intravenoso de infecçóes por MRSA em pacientes graves, clinicamente instáveis (Frymoyer, Hersh et al. 2009) $\mathrm{H}<$ style face $=$ "smallcaps" $>$ ersh $</$ style $><$ style face="italic" $>$ et al. $</$ style $>2009$, porém casos de resistência ao mesmo já são relatados com MIC variando entre 8 a $16 \mathrm{mg} / \mathrm{mL}$ desde 1997, e a heterogeneidade de resistência pode ser um dos fatores das falhas no tratamento de infecçóes invasivas por MRSA(Climo, PAtron et al. 1999; Frymoyer, Hersh et al. 2009)P<style face=" smallcaps" $>$ atron $</$ style $><$ style face $=$ "italic" $>$ et al. $<$ l style $>1999 ; \mathrm{F}<$ style face $=$ "smallcaps" $>$ rymoyer $</$ style $>$, $\mathrm{H}<$ style face="smallcaps" $>$ ersh $</$ style $><$ style face="italic" $>$ et al.</style $>2009$.

Para um sucesso no tratamento, a maior parte dos casos inclui uma combinação de abordagens terapêuticas, incluindo cirurgia em casos que se apresentam como abscessos (Climo, Patron et al. 1999). A combinação de glicopeptídeos e beta-lactâmicos como oxacilina pode causar um 
sinergismo positivo no efeito antimicrobiano para MRSA, uma vez que atuam em diferentes estágios na parede celular, sendo uma das abordagens terapêuticas no caso de infecção por MRSA com susceptibilidade reduzida a vancomicina (Climo, Patron et al. 1999; Fox, Lampen et al. 2006). Em casos de falha do tratamento com a vancomicina em pacientes adultos, deve-se fazer uma busca de outros focos de infecção, drenagem ou desbridamento cirúrgico. Novos protótipos terapêuticos estão sendo utilizados como daptomicina, rifampicina, linezolida em altas doses e posologias específicas (Frymoyer, Hersh et al. 2009; Liu, Bayer et al. 2011; Hagihara, Umemura et al. 2012).

Daptomicina é uma droga lipoglicopeptídica utilizada via intravenosa (Vilhena and Bettencourt 2012) com ação bactericida para MRSA e para outras bactérias Gram-positivas no tratamento de bacteremia, endocardite do lado direito e infecçôes de pele e tecidos moles. Deve seguir o esquema posológico de $10 \mathrm{mg} / \mathrm{Kg} /$ dia quando isolado ou em combinação com outro medicamento como a gentamicina por exemplo (Liu, BAYER et al. 2011). Se utilizada como primeira linha de tratamento, apresenta maiores resultados benéficos quando comparada a infecções previamente tratadas por vancomicina (GONZALEZ-RuIZ, BEIRASFernandez et al. 2011; Hagihara, UMEMura et al. 2012; Vilhena and Bettencourt 2012). Diferente da linezolida, a daptomicina é bactericida, e ao contrário da vancomicina apresenta semelhante atividade contra isolados de MRSA e MSSA. (Gonzalez-Ruiz, Beiras-Fernandez et al. 2011). O mecanismo de ação da daptomicina ainda não está bem descrito, porém sabe-se que sua ação bactericida se dá por uma desestruturação na membrana celular da célula bacteriana, diferente da maioria dos antimicrobianos existentes. A daptomicina demonstra baixos efeitos adversos quando comparada a outros antimicrobianos (VILHENA and BETTENCOURT 2012).

A linezolida é um medicamento com 100\% de biodisponibilidade por via oral, desta forma a utilização por via intravenosa somente é recomendada quando houver contraindicação ao uso do trato gastrointestinal, como por exemplo, no caso íleo paralítico, com instabilidade clínica devido à sepse grave. Apresenta atividade contra MSSA, MRSA e estreptococos (Elliott, Zaoutis et al. 2009; GRIffin, Peyrani et al. 2012; Segarra-Newnham and ChurCH 2012). Tem boa penetração nos tecidos, náo precisa de monitoração terapêutica e pode ser usada via intravenosa ou via oral. Quando administrado por via intravenosa, a posologia para adultos é de $600 \mathrm{mg}$ duas vezes ao dia. A resistência a linezolida é rara (LiU, BAYER et al. 2011). O principal evento adverso associado ao tratamento com linezolida é a mielossupressão reversível, na maior parte com trombocitopenia, o que usualmente ocorre após 14 dias de tratamento com esse fármaco (Gonzalez-Ruiz, BeirasFernandez et al. 2011; Hagihara, Umemura et al. 2012).

A teicoplanina é um glicopeptídeo com espectro de ação e mecanismo semelhante a vancomicina. Tal fármaco tem tempo de meia vida maior que o da vancomicina, porém tem menor taxa de penetração no tecido. A vantagem do uso da teicoplanina é que apresenta menor efeito nefrotóxico do que a vancomicina, além do fato de poder ser utilizada por via intramuscular (B. 2011; Hagihara, UMEMURA et al. 2012; SEKI, YABUno et al. 2012).

Clindamicina é indicada e aprovada pelo FDA no tratamento de infecçóes graves por $S$. aureus e não especificamente para infecçóes por MRSA, porém vem sendo utilizada em larga escala para tratar infecçôes por CA-MRSA, Staphylococcus aureus Resistentes a Meticilina associados a infecçôes de ambiente comunitário, em crianças. Possui ação bacteriostática, não sendo recomendada para infecçóes endovasculares. Apresenta boa penetração nos tecidos. O efeito adverso mais relatado é a diarreia. A posologia indicada para tratar infecçôes de pele e tecido mole é $600 \mathrm{mg}$ por três vezes ao dia por via intravenosa ou via oral (LIU, BAYER et al. 2011; Webber, Federinko et al. 2013; Zaman 2013).

Telavancina é um lipoglicopeptídeo bactericida usado contra MRSA contra Staphylococcus aureus com resistência intermediaria e resistência a vancomicina, respectivamente, VISA e VRSA. A posologia indicada é de $10 \mathrm{mg} / \mathrm{kg}$ uma vez ao dia via intravenosa e o efeito adverso mais característico é a nefrotoxicidade, porém não se encontra disponível ainda nos EUA(Segarra-Newnham and Church 2012; Amanatullah, Matthew J. Lopez B. S et al. 2013).

A rifampicina tem atividade bactericida contra $S$. aureus, obtém elevados níveis celulares e é capaz de penetrar em biofilmes. Para evitar resistência, deve-se utilizar combinada a outro medicamento igualmente eficaz. A posologia de $600 \mathrm{mg} /$ dia via oral ou via intravenosa no tratamento de infecçôes por MRSA é adotada, porém sua combinação com outros medicamentos ainda não é bem estabelecida (LiU, BAYER et al. 2011).

As tetraciclinas são indicadas para tratar infecçóes de pele e tecidos moles por $S$. aureus, não necessariamente exclusivas para MRSA (Liu, BAYer et al. 2011; Webber, FeDERINKO et al. 2013). 


\section{CONCLUSÃO}

S. aureus é um importante patógeno associado com infecçóes que podem variar em gravidade, sendo relacionadas aos ambientes hospitalar e comunitário. Caso não sejam precoce e adequadamente abordadas, tais infecçóes ocasionam morbidade e mortalidade significativas ao paciente. Desta forma, é necessário o entendimento da terapêutica precisa adotada para cada caso específico, levando-se em consideraçáo o local da infecçáa, a gravidade do quadro clínico e as particularidades do paciente (co-morbidades como insuficiência renal, por exemplo), além de características genéticas de resistência. $\mathrm{O}$ uso indiscriminado de antimicrobianos deve ser evitado, na tentativa de se reduzir novos casos de resistência, e a monitoração dos níveis terapêuticos dos fármacos deve ser realiada sempre que possível, a fim de evitar graves efeitos adversos. 


\section{REFERÊNCIAS}

1. (CDC), C. F. D. C. A. P. Vancomycin-resistant Staphylococcus aureus--Pennsylvania, 2002: MMWR Morb Mortal Wkly Rep 2002 Oct 18;51(41):931., 51(41), 931, 2002.

2. AMANATULLAH, D. F.; MATTHEW J. LOPEZ B. $S$, et al. Artificial Elevation of Prothrombin Time by Telavancin: Clin Orthop Relat Res, 471 332-335, 2013.

3. ARCHER, N. K.; MAZAITIS, M. J., et al. Staphylococcus aureus biofilms: properties, regulation, and roles in human disease: Virulence, 2(5), 445-459, 2011.

B., S. Staphylococcus aureus and antibiotic resistance: Mikrobiyol Bul, 45(3), 565-576, 2011.

4. ClimO, M. W.; PATRON, R. L., et al. Combinations of Vancomycin and b-Lactams Are Synergistic against Staphylococci with Reduced Susceptibilities to Vancomycin: Antimicrobial Agentes and Chemotherapy, 43(7), 1747-1753, 1999.

5. DA COSTA, T. M.; PINHeirO, M. G., et al. Características clínicas e Esquema de Tratamento Medicamentoso de Infecçóes por Staphylococcus aureus: uma possível proposta de identificação para o laboratorista e tratamento para o clínico: Praxis, 52011.

6. DERESINSKI, S. Methicillin-resistant Staphylococcus aureus: an evolutionary, epidemiologic, and therapeutic odyssey: Clin Infect Dis, 40(4), 562-573, 2005.

7. ELLIOTT, D. J.; ZAOUTIS, T. E., et al. Empiric Antimicrobial Therapy for Pediatric Skin and Soft-Tissue Infections in the Era of Methicillin-Resistant Staphylococcus aureus: PEDIATRICS, 123(6), e959966, 2009.

8. FALORD, M.; MADER, U., et al. Investigation of the Staphylococcus aureus GraSR regulon reveals novel links to virulence, stress response and cell wall signal transduction pathways: Plos One, 6(7), 21323, 2011.

9. FOX, P. M.; LAMPEN, R. J., et al. Successful Therapy of Experimental Endocarditis Caused by Vancomycin-Resistant Staphylococcus aureus with a Combination of Vancomycin and B-Lactam Antibiotics: Antimicrobial Agentes and Chemotherapy, 50(9), 2951-2956, 2006.
10. FRYMOYER, A.; HERSH, A. D., et al. Current Recommended Dosing of Vancomycin for Children With Invasive Methicillin-Resistant Staphylococcus aureus Infections Is Inadequate: The Pediatric Infectious Disease Journal, 28(5), 398-402, 2009.

11. GIAROLA, L. B.; SANTOS, R. R., et al. HLA molecules and nasal carriage of Staphylococcus aureus isolated from dialysis and kidney transplant patients at a hospital in Southern Brazil: BMC Res Notes, 5 90, 2012.

12. GONZALEZ-RUIZ, A.; BEIRAS-FERNANDEZ, A., et al. Clinical experience with daptomycin in Europe: the first 2.5 years: J Antimicrob Chemother, 1-8, 2011.

13. GRIFFIN, A. T.; PEYRANI, P., et al. Empiric therapy directed against MRSA in patients admitted to the intensive care unit does not improve outcomes in community-acquired pneumonia: Infection and Immunity, 2012.

14. HAGIHARA, M.; UMEMURA, T., et al. Daptomycin approved in Japan for the treatment of methicillinresistant Staphylococcus aureus: Ther Clin Risk Manag, 8 79-86, 2012.

15. HIRAMATSU, K.; HANAKI, H., et al. Methicillin-resistant Staphylococcus aureus clinical strain with reduced vancomycin susceptibility: J Antimicrob Chemother, 40 135-136, 1997.

16. IPPOLITO, G.; LEONE, S., et al. Methicillin-resistant Staphylococcus aureus: the superbug: Int J Infect Dis, 14 (4), S7-11, 2010.

17. KATEETE, D. P.; KIMANI, C. N., et al. Identification of Staphylococcus aureus: DNase and Mannitol salt agar improve the efficiency of the tube coagulase test: Ann Clin Microbiol Antimicrob, 9 23, 2010.

LINDSAY, J. A. and HOLDEN, M. T. Staphylococcus aureus: superbug, super genome?: Trends Microbiol, 12(8), 378-385, 2004.

18. LIU, C.; BAYER, A., et al. Clinical Practice Guidelines by the Infectious Diseases Society of America for the Treatment of Methicillin-Resistant Staphylococcus Aureus Infections in Adults and Children: Clinical Infectious Diseases, 1-38, 2011.

19. LUNA, C. M.; RODRIGUEZ-NORIEGA, E., et al. [Treatment of methicillin-resistant Staphylococcus au- 
reus (MRSA) infections in Latin America]: Rev Chilena Infectol, 27((2)), 94-103, 2010.

20. MURRAY, P. R.; BARON, E. J., et al. (2003). Staphylococcus; Micrococcus; and Other Catalase-Positive Cocci that Grow Aerobically.

21. PARK, S. Y.; KIM, S. M., et al. The Prevalence, Genotype and Antimicrobial Susceptibility of High- and Low-Level Mupirocin Resistant Methicillin-Resistant Staphylococcus aureus: Ann Dermatol, 24(1), 32-38, 2012.

22. RAMMELKAMP, C. H. and MAXON, T. Resistance of Staphylococcus aureus to the Action of Penicillin: Proc Roy Soc Exper Biol Med, 51 386-389, 1942.

23. RODRIGUEZ-NORIEGA, E. and SEAS, C. The changing pattern of methicillin-resistant staphylococcus aureus clones in Latin America: implications for clinical practice in the region: Braz J Infect Dis, $14 \mathrm{Su}$ ppl 2 S87-96, 2010

24. RUDKIN, J. K.; EDWARDS, A. M., et al. Methicillin resistance reduces the virulence of healthcare-associated methicillin-resistant Staphylococcus aureus by interfering with the agr quorum sensing system: I Infect Dis, 205(5), 798-806, 2012.

25. SANTOS, A. L.; SANTOS, D. O., et al. Staphylococcus aureus: visiting a strain of clinical importance: $J$ Bras Patol Med Lab, 43(6), 413-423, 2007.

26. SEGARRA-NEWNHAM, M. and CHURCH, T. J. Pharmacotherapy for methicillin-resistant Staphylococcus aureus nosocomial pneumonia: Ann Pharmacother, 46(12), 1678-1687, 2012.

27. SEKI, M.; YABUNO, K., et al. Loading regimen required to rapidly achieve therapeutic trough plasma concentration of teicoplanin and evaluation of clinical features: Clinical Pharmacology: Advances and Applications, 4 71-75, 2012.

28. SHITTU AO; UDO EE, et al. Phenotypic and molecular characterization of Staphylococcus aureus isolates expressing low- and high-level mupirocin resistance in Nigeria and South Africa: BMC Infect Dis, 28 9-10, 2009.

29. VILHENA, C. and BETTENCOURT, A. Daptomycin: A Review of Properties, Clinical Use, Drug Deli- very and Resistance: Medicinal Chemistry, 12 202-209, 2012.

30. WEBBER, B. J.; FEDERINKO, S. P., et al. Staphylococcus aureus and other skin and soft tissue infections among basic military trainees, Lackland Air Force Base, Texas, 2008-2012.: MSMR, 20(1), 12-15, 2013.

31. YOUNG, B. C.; GOLUBCHIK, T., et al. Evolutionary dynamics of Staphylococcus aureus during progression from carriage to disease: Proc Natl Acad Sci US A, 109(12), 4550-4555, 2012.

32. ZAMAN, S. R. MRSA infected emphysematous bullae in an asymptomatic COPD patient: BMJ Case Rep, 2013. 